\title{
Álcool e Violência em Homens e Mulheres
}

\author{
Alcohol: Gender and Implications in the Violence
}

\author{
Rosa Maria Martins de Almeida*, Graciela Gema Pasa \& Morgana Scheffer \\ Universidade do Vale do Rio dos Sinos
}

\begin{abstract}
Resumo
O uso nocivo do álcool configura-se como um problema de saúde pública, associado ao aumento da violência, envolvendo ambos os sexos. Esse artigo tem por objetivo discutir sobre o impacto do uso do álcool em homens e mulheres do ponto de vista neurobiológico, enfatizando a ação psicoativa da substância e sua implicação no comportamento violento. Foi conduzida uma análise baseada em artigos selecionados nas fontes eletrônicas do Scielo, LILACS, MEDLINE, PubMed e Web of Science no período de 1996 a 2008. Do total de 420 artigos selecionados, 90 foram considerados relevantes para a análise. Verificou-se que o uso nocivo do álcool causa mudanças neuroquímicas e alterações nas funções cognitivas, podendo gerar comportamentos violentos em homens e mulheres, entretanto, evidenciou-se importantes diferenças entre os sexos quanto à ação psicoativa do álcool, assim como, no tipo de violência expressa. Estudos sobre a temática proposta ainda são escassos, sugerindo a necessidade de pesquisas futuras que possam contribuir para um melhor entendimento e para ações preventivas eficazes.

Palavras-chaves: Álcool; Violência; Cérebro; Cognição; Comportamento.
\end{abstract}

\begin{abstract}
The abuse of alcohol can engender serious public health problems in certain people, particularly due to its link to violence involving both men and women. This article has the aim to discuss the impact of alcohol in men and women regarding neurobiological mechanisms, emphasizing its psychoactive effects as well as its implication for violent behavior. An analysis was conduct based on reviews and articles in electronic databases, selected from 1996 to 2008 at Scielo, Lilacs, MEDLINE, Pub Med and Web of Science. From a total of 420 selected articles 90 were considered relevant for this analysis. It was evident that the abuse of alcohol causes changes in neurochemistry and in cognitive functions, and some of those changes lead to violent behavior in men and women. However, there are important differences between both genders and the type of aggressive behavior expressed. Studies on this topic are still rare and more research is necessary in order to develop better diagnostic tools and favor relevant neurobiological mechanisms for more effective treatments. Keywords: Alcohol; Violence; Brain; Cognition; Behavior.
\end{abstract}

O uso nocivo e crônico do álcool configura-se como grave problema de saúde pública, tratado como uma questão de ordem internacional (Dick \& Bierut, 2006; Fontes, Figlie, \& Laranjeira, 2006; Liu \& Wuerker, 2005; Marques \& Cruz, 2000; Oscar-Berman, Shagrin, Evert, \& Epstein, 1997; Pettinati \& Rabinowitz, 2005) tanto por estar diretamente relacionado ao significativo aumento da violência e criminalidade (Chalub \& Telles, 2006; Chermack \& Giancola, 1997; Martin \& Bryant, 2001; Pillon, O`Brien, \& Chavez, 2005), como, também, por ser causa direta de morbidade e mortalidade na população em geral (Fontes et al., 2006; Klatsky, Marín-León, Oliveira, \& Botega, 2007; Meloni \& Laranjeira, 2004; Nakahara et al., 2002; OscarBerman et al., 1997). Dados demográficos brasileiros revelam que o consumo do álcool determina mais de $10 \%$ de toda a morbidade e mortalidade ocorrida no país sendo,

* Endereço para correspondência: Universidade do Vale do Rio dos Sinos, Laboratório de Neurociências, Centro 2, Av. Unisinos, 950, São Leopoldo, RS, Brasil, CEP 93022-000. E-mail:rmalmeida@unisinos.br desta forma, o problema público mais grave (Meloni \& Laranjeira, 2004).

Ao longo dos anos, o consumo do álcool vem aumentando substancialmente e acarretando importantes prejuízos tanto individuais quanto sociais (Cunha \& Novaes, 2004; Dick \& Bierut, 2006). Segundo a Organização Mundial da Saúde ([OMS], 2004), estima-se que existam aproximadamente dois bilhões de pessoas no mundo consumidoras de bebidas alcoólicas, sendo 76,3 milhões as que apresentam problemas com o uso do álcool, o que representam índices alarmantes.

Por ser de baixo custo, de fácil acesso e de grande aceitabilidade social, o álcool torna-se uma das substâncias psicoativas mais precocemente consumidas no mundo (Pechansky, Szobot, \& Scivoletto, 2004; Pillon et al., 2005; Siqueira, 2004). Este fato resulta no aumento de consumidores jovens, contribuindo para a mortalidade precoce (Kirkcaldy, Siefen, Urkin, \& Merrick, 2006; Nobrega \& Oliveira, 2005) e para maior exposição ao risco de desenvolver problemas futuros relacionados ao uso da substância (Chermack, Wryobeck, Walton, \& Blow, 2006; Grant 
et al., 2006; Marques \& Cruz, 2000; Ramisetty-Mikler, Goebert, Nishimura, \& Caetano, 2006).

O álcool também aparece associado com o uso de outras substâncias lícitas, como o tabaco (Edelstein, KritzSilverstein, \& Barrett-Connor, 1998; Enoch, 2003; Roy, Parker, Mitchell, \& Wilhelm, 2001) e/ou ilícitas, como a maconha (Grant et al., 2006; Pillon et al., 2005) e cocaína (Martin \& Bryant, 2001), sendo considerado como preconizador no uso destas substâncias (Grant et al., 2006). O uso do álcool associado a outras drogas resulta em níveis muito elevados de agressividade, constituindo-se como um importante fator gerador de violência (Martin \& Bryant, 2001).

Dentre os aspectos envolvidos com o uso do álcool, verifica-se importante associação com o meio social favorável ao uso (Parker \& Auerhahn, 1998), isto é, onde a prática do beber é considerada comum; classe sócio-econômica e nível de escolaridade baixos; histórico familiar de alcoolismo (Marques \& Cruz, 2000); predisposição genética (Dick \& Bierut, 2006; Enoch, 2003), pobre relacionamento com os pais, alta prevalência de transtornos mentais e violência (Marín-León et al., 2007; Meloni \& Laranjeira, 2004). Desta forma, considera-se o uso do álcool como um problema multi-fatorial, de difícil controle e tratamento.

A violência decorrente do uso do álcool está relacionada principalmente pelas propriedades psicoativas da substância. Do ponto de vista neurobiológico, alguns efeitos do uso nocivo e crônico do álcool - inclui distorção cognitiva e de percepção, déficit de atenção, julgamento errado de uma situação, mudanças neuroquímicas, prejuízo de funcionamento neuropsicológico ou associação com transtornos de personalidade - originam ou estimulam comportamentos violentos (Laranjeira, 2004).

Embora haja maior prevalência de indivíduos do sexo masculino nas pesquisas envolvendo o uso do álcool e violência, percebe-se um aumento importante de estudos voltados ao sexo feminino, enfatizando sua maior vulnerabilidade à dependência química e outras patologias, bem como, histórico de violência doméstica e grave risco de suicídio, associados ao uso da substância (Chalub \& Telles, 2006; Galera, Roldán, \& O’Brien, 2005; Nobrega \& Oliveira, 2005; Simão, Kerr-Corrêa, Dalben, \& Smaira, 2002; Siqueira, 2004).

A partir de estudos com a população feminina, verificou-se que a metabolização do álcool e de outras substâncias psicoativas ocorrem de maneira mais lenta nas mulheres do que nos homens, isso se deve ao fato da mulher apresentar na sua composição corpórea menos água e maior quantidade de tecido gorduroso (Nobrega \& Oliveira, 2005). Assim, elas se tornam mais susceptíveis aos prejuízos associados ao seu consumo, mesmo ingerindo níveis mais baixos de álcool por período mais curto (HernandézÁvila, Rounsaville, \& Kranzler, 2004). Dentre osprincipais problemas decorrentes do uso para a mulher incluem miocardiopatia, miopatia e lesão cerebral; hepatite alcoólica quase sempre progride para cirrose, inibição da ovulação, diminuição da fertilidade e vários problemas ginecológicos e obstétricos (Nobrega \& Oli-veira, 2005).
Uma vez que há uma maior vulnerabilidade do sexo feminino em relação aos efeitos psicoativos do álcool, bem como, maior impacto (neuro)biológico se comparado ao sexo masculino representando importantes diferenças entre os sexos, faz-se pertinente tecer comparações quanto à ação psicoativa do álcool também em relação ao comportamento agressivo e/ou violento em homens e mulheres para um melhor entendimento do fenômeno (Brady \& Randall, 1999; Cha, Li, Wilson, \& Swartzwelder, 2006; Epstein, Fischer-Elber, \& Al-Otaiba, 2007; Glazkov \& Sviderskaia, 2006; Hommer, 2003; Nobrega, \& Oliveira, 2005).

Tendo em vista o exposto, este artigo foi desenvolvido com o objetivo de discutir sobre o impacto do uso nocivo e crônico do álcool em homens e mulheres do ponto de vista neurobiológico, enfatizando a ação psicoativa da substância e sua implicação no comportamento violento.

A composição do presente artigo resultou de uma análise baseada em artigos selecionados nas fontes eletrônicas do Scielo, LILACS, MEDLINE, Science Direct, PubMed e Web of Science no período de 1996 a 2008 a partir das seguintes palavras-chave: álcool (alcohol); violência (violence); agressividade (aggessivity); homem e mulher (man and woman). cognição (cognition); cérebro (brain). Todo o material coletado, que constou de 420 artigos foi devidamente, analisado, comparado e avaliado quanto a sua contribuição para o objetivo do estudo, sendo considerados relevantes o total de 90 artigos. Na medida em que o material foi analisado tornou-se claro e evidente que esse tema é ainda pouco explorado e necessita de mais estudos.

\section{Uso Nocivo e Crônico do Álcool e sua Relação com a Violência}

O uso nocivo do álcool é um dos principais responsáveis pela maioria dos casos de violência envolvendo o sexo masculino e feminino, (Badawy, 2003; Borders, Barnwell, \& Earleywine, 2007; Corrigan \& Watson, 2005; Johansson, Bergvall, \& Hansen, 1999; Martin \& Bryant, 2001; Parker \& Auerhahn, 1998; Sher, 2006; Thompson \& Kingree, 2004; Zilberman \& Blume, 2005) contribuindo para tornálo, nos dias atuais, um dos principais problemas de saúde pública (Liu \& Wuerker, 2005). O risco da associação do álcool com a violência não ocorrem apenas com os bebedores nocivos e regulares, mas também entre bebedores moderados ou eventuais (Rabello \& Caldas Junior, 2007).

Em geral, a violência decorrente do uso do álcool é associada, na maior parte dos estudos sobre o tema, ao sexo masculino como o agressor (Abrahams, Jewkes, Laubscher, \& Hoffman, 2006; Adeodato, Carvalho, Siqueira, \& Souza, 2005; Hasegawa, Bessho, Hosoya, \& Deguchi, 2005; Thompson \& Kingree, 2004). Diante disso, deve-se considerar o fato de que são os homens quem mais utilizam esta substância. Também é importante considerar que o álcool age sobre o organismo de homens e mulheres de maneira distinta (Devaud, Alele, \& Ritu).

No sexo masculino, a violência associada ao uso do álcool é comumente identificada através de comportamentos agressivos direcionados ao próprio indivíduo ou a 
outros, o que acarreta prejuízos, muitas vezes, irreparáveis (Zilberman \& Blume, 2005).

A autoviolência é retratada através de exposição moral; comportamentos de risco como: sexo desprotegido, acidentes de trânsito devido ao dirigir alcoolizado e em alta velocidade e tentativas de suicídio (Conner et al., 2001; Laranjeira \& Romano, 2004; Sher, 2006). A violência direcionada aos outros, por sua vez, envolve, principalmente familiares mais próximos, como filhos e cônjuge, cometida e vivenciada de diversas formas: violência moral e sexual, espancamento, humilhações verbais, homicídio (Abrahams et al., 2006; Hasegawa et al., 2005; Thompson \& Kingree, 2004; Zilberman \& Blume, 2005), geralmente, os episódios de violência são repetitivos e progressivamente mais graves, caracterizando-se por situações de cronicidade e crescente periodicidade (Vaiz Bonifaz \& Nakano, 2004). Dentre as substâncias psicoativas, o álcool torna-se a substância mais nociva para o funcionamento familiar (Rabello \& Caldas Junior, 2007).

O uso do álcool pelo companheiro está presente em $70 \%$ dos casos de mulheres violentadas que procuram ajuda, além disso, verifica-se correlação direta entre consumo de álcool pelo agressor e agressão aos filhos (Adeodato et al., 2005). Há evidência de que indivíduos violentos, de ambos os sexos, geralmente apresentam história de violência na infância (Chermack et al., 2006).

Nas mulheres, o uso do álcool associado à violência aparece, com maior prevalência, caracterizado por comportamentos auto-agressivos, através de tentativas de suicídio, bem como, de suicídio terminado (Conner et al., 2001; Pillay, Van der Veen, \& Wassenaar, 2001). Além disso, muitas vezes, o ato de beber passa a fazer parte do dia-adia de mulheres, cujos cônjuges alcoolizados ou não utilizam a violência na relação. Dessa forma, intensifica-se tanto a violência, quanto o consumo do álcool, os quais afetam a saúde física e mental (Zilberman \& Blume, 2005). Quadros de depressão, ansiedade e desordem de estresse pós-traumático, mudanças no sistema endócrino, aumento do uso de álcool e drogas entre mulheres que procuram atenção médica podem sugerir uma história de violência doméstica (Augengraun, Wilson, \& Allister, 2001).

Corroborando estes dados, constatou-se em uma pesquisa realizada no México, que mulheres que procuravam atendimento nos serviços voltados à saúde pública com queixa de ansiedade e depressão, tinham como principais fatores associados o uso nocivo do álcool e história de violência doméstica perpetrada pelo marido (Valdez-Santiago et al., 2006).

A violência contra a mulher, cometida na maioria das vezes pelos próprios parceiros, envolve usuários regulares e/ou eventuais do álcool, sendo que as agressões ocorrem geralmente quando o agressor está sob o efeito do álcool. Dentre os tipos de violência sofrida pelas mulheres, destacam-se as violências psicológicas, físicas e sexuais (Adeodato et al., 2005).

A violência psicológica caracterizada por ameaças e humilhações verbais é a mais freqüente e identificada pelas mulheres, seguida pela violência física e sexual. Res- salta-se que a violência sexual, embora menos presente nos relatos das mulheres vitimizadas pode ser, muitas vezes, confundida e mascarada pela relação sexual, e é a que causa danos mais significativos na saúde da mulher (Ramos-Lira, Saltijeral-Méndez, Romero-Mendoza, Caballero-Gutiérrez, \& Martínez-Vélez, 2001). Conseqüências não-mortais da violência por parceiros incluem efeitos permanentes na auto-estima e auto-imagem, deixando-as com menos possibilidade de se proteger, menos seguras do seu valor, e mais propensas a aceitar a vitimização como sendo parte de sua condição de mulher (Adeodato et al., 2005).

A violência sexual está diretamente associada a maiores índices de suicídio, abuso de álcool, outras drogas, sofrimento psíquico em geral e a outros problemas como cefaléia e distúrbios gastrointestinais. Além disso, trazem considerações importantes em relação à saúde reprodutiva: a violência contra a mulher tem sido associada às dores pélvicas crônicas, às doenças sexualmente transmissíveis, como a síndrome da imunodeficiência humana adquirida (Aids), além de doenças pélvicas inflamatórias e gravidez indesejada (Schraiber, D’Oliveira, França-Junior, \& Pinho, 2002).

É importante salientar que o uso do álcool não se limita à violência doméstica, ele também assume um lugar na sociedade, tendo influência importante no aumento da criminalidade, vandalismo, desordem pública acarretando significativos custos sociais (Dick \& Bierut, 2006; Martin \& Bryant, 2001). Em um estudo realizado na Europa, com uma amostra de 415 prisioneiros, de ambos os sexos, com histórico de crimes praticados (homicídios, agressão, roubos) verificou-se alta prevalência do uso nocivo do álcool principalmente entre o sexo masculino (Watzke, Ullrich, \& Marneros, 2006).

Entre os adolescentes o uso do álcool aparece associado a comportamentos de risco em ambos os sexos. Entretanto, maior prevalência foi observada entre o sexo masculino, principalmente, através de tentativas de suicídio e comportamento sexual desprotegido (Bonomo et al., 2001; Kirkcaldy et al., 2006; Mack \& Frances, 2003; Pechansky et al., 2004). Em uma amostra de 3872 adolescentes de ambos os sexos, que aqueles que tinham usado o álcool mais de vinte vezes na vida tinham duas vezes maior probabilidade de desenvolver comportamentos violentos daqueles que nunca tinham feito uso da substância (Gudlaugsdottir, Vilhjalmsson, Kristjansdottir, Jacobsen, \& Meyrowitsch, 2004). Comprovando assim, o impacto que o uso do álcool tem no desenvolvimento de práticas violentas, também, entre os jovens.

Considerando que o uso nocivo do álcool, na maioria dos casos, é iniciado ainda na adolescência, momento onde o adolescente está se estruturando em termos biológicos, sociais, pessoais e emocionais, seu impacto sobre a neuroquímica cerebral resultará em pior ajustamento social e no retardo do desenvolvimento de suas habilidades, ocasionando em prejuízos que o acompanharão ao longo da vida (Chavez, O`Brien, \& Pillon, 2005; Marques \& Cruz, 2000; Pechansky et al., 2004). De acordo com isso, constatou-se em um estudo prévio, que adolescentes com uso nocivo de 
álcool têm sete vezes mais chance de desenvolver Transtorno de Conduta, duas vezes mais Transtorno Opositivo Desafiante, duas vezes mais Transtorno de Déficit de Atenção e Hiperatividade (TDAH) e três vezes mais Transtornos Depressivos comparados aos jovens não usuários (Clark, Lynch, Donovan, \& Block, 2001).

\section{Ação do Álcool no Sistema Nervoso Central e seus Efeitos Psicoativos}

O álcool tem ação no Sistema Nervoso Central (SNC) e causa alterações orgânicas, cognitivas e comportamentais em ambos os sexos (Devaud et al., 2003; Saletu-Zyhlarz et al., 2004). Conforme o tempo de uso e a quantidade consumida, graves prejuízos à saúde podem se intensificar e tornar-se irreversíveis, impactando negativamente na qualidade de vida (Cunha \& Novaes, 2004; Oscar-Berman et al., 1997).

Sua ação difusa no SNC altera as funções neuroquímicas dos sistemas: GABA (ácido gama-aminobutírico) (Cha et al., 2006), glutamato (Oscar-Berman et al., 1997), serotonina (Badawy, 2003; Enoch, 2003; Lavine, 1997), norepinefrina (Johansson et al., 1999; Lavine, 1997), dopamina e acetilcolina (Dick \& Bierut, 2006; Eckardt et al., 1998). O álcool pode afetar estes sistemas diretamente e/ou as interações entre eles (Eckardt et al., 1998), comprometendo o funcionamento de diversas áreas cerebrais de homens e mulheres (Mann et al., 2005).

No uso nocivo do álcool, caracterizado pela intoxicação, verificam-se prejuízos cognitivos pouco acentuados, entretanto, sua ação desencadeia importante ativação em várias áreas do cérebro, sendo o giro do cíngulo a região mais afetada (Amen, Stubblefield, Carmicheal, \& Thisted, 1999). O uso nocivo do álcool tende a comprometer a atenção, a memória, as funções executivas e as viso-espaciais, provoca alteração no comportamento, como: desinibição, aumento da agressividade, perda do controle dos impulsos, euforia, associado à exposição ao risco como: acidentes de carro; homicídios; suicídios; quedas; queimaduras e afogamento (Cunha \& Novaes, 2004; Laranjeira \& Romano, 2004; Marín-León et al., 2007; Marques \& Cruz, 2000).

No uso crônico, por sua vez, técnicas de neuro-imagem fornecem evidências importantes de alterações físicas do cérebro, tais como: atrofia de fibras nervosas e perdas de substância cinzenta e branca (Nakahara et al., 2002; Pfefferbaum, Adalsteinsson, \& Sullivan, 2006). As alterações cerebrais causadas pelo uso crônico do álcool ocasionam importantes danos em diferentes regiões como: córtex pré-frontal (Brun \& Andersson, 2001; Schweinsburg et al., 2003), temporal, parietal e occipital, sistema límbico, incluindo amígdala e hipocampo (Bartels et al., 2007); hipotálamo e corpo caloso (Oscar-Berman et al., 1997).

Em uma amostra constituída por 50 homens e 10 mulheres em tratamento para o uso do álcool constatou-se que $60 \%$ apresentaram alterações cerebrais mesmo depois de 28 dias de abstinência. As áreas mais afetadas foram: os lobos pré-frontal, temporal e parietal, de uma maneira ge- ral, constataram-se alterações focais em 58,3\%, difusas em $41,7 \%$ e bilateral em $95 \%$, comprovando que o uso crônico acarreta prejuízos importantes em ambos os sexos, mesmo após período de abstinência (Pallavicini et al., 2002).

As alterações neuroquímicas e os danos cerebrais ocasionados pelo uso crônico do álcool provocam importantes prejuízos das funções cognitivas e executivas em ambos os sexos (Brown, Tapert, \& Brown, 2001; Cunha \& Novaes, 2004) observados na memória; (Brown, Tapert, Granholm, \& Delis, 2000; Pechansky et al., 2004; Rosenbloom, O'Reilly, Sassoon, Sullivan, \& Pfefferbaum, 2005) na atenção, nas habilidades viso-espaciais, no raciocínio abstrato; na aquisição de informações (Bates, 1997; Cunha \& Novaes, 2004; Oscar-Berman et al., 1997), no controle dos impulsos (Mitchell, Fields, D'Esposito, \& Boettiger, 2005) e nos níveis de ansiedade e agressividade. Estimativas indicam que dentre os usuários crônicos do álcool, 50 a $85 \%$ exibem sinais de prejuízos cognitivos (Oscar-Berman et al., 1997).

Corroborando estes dados, Monnot, Nixon, Lovallo e Ross (2001) verificaram que usuários crônicos do álcool apresentam danos nas habilidades associadas com o hemisfério direito, as quais estão relacionadas com aspectos afetivos e emocionais. Desta forma, constata-se que em usuários do álcool, a capacidade de compreender a emoção associada à linguagem está prejudicada, resultando em erros de julgamento e, conseqüentemente, perdas importantes nas interações sociais. Os autores ressaltam que a perda da capacidade de julgamento e dificuldade de interpretar o significado da linguagem, associados à agressividade resultante da ação psicoativa do álcool, são importantes geradores de violência.

As alterações cerebrais decorrentes do consumo crônico de álcool podem chegar a estágios muito avançados de deterioração mental, como no caso da Demência Persistente Induzida pelo Álcool e do Transtorno Amnéstico Persistente induzido pelo Álcool (Síndrome de Korsakoff) (Cunha \& Novaes, 2004). O uso crônico do álcool também é responsável por desencadear outras patologias como: neoplasias de lábio, cavidade oral, faringe, laringe, esôfago e fígado, gastrite, varizes esofagianas, pancreatites aguda e crônica, diabetes mellitus, tuberculose, hepatite $\mathrm{C}$, pneumonia e convulsões, as quais, prejudicam de forma considerável a qualidade de vida dessas pessoas (Marín-León et al., 2007).

Técnicas de neuro-imagem estudam os danos cerebrais causados ou envolvidos com o uso nocivo e crônico do álcool, mas ainda são poucas as pesquisas que estudam as diferenças existentes entre os sexos (Mann et al., 2005; Prendergast, 2004) e as implicações no comportamento violento. Por muitos anos, os investigadores evitaram incluir o sexo feminino em suas pesquisas devido a pouca compreensão a respeito das influências do ciclo hormonal. Entretanto, com o passar do tempo, a comunidade científica tornou-se cada vez mais ciente da importância de estudar os efeitos do álcool também nas mulheres, considerando sua maior vulnerabilidade (Devaud et al., 2003).

A partir de modelos animais, Devaud et al. (2003) ve- 
rificaram que ratas fêmeas, após a retirada do álcool, respondiam melhor à abstinência do que ratos machos. Além disso, com a retirada do álcool pôde-se verificar alterações funcionais do receptor GABA mais significativamente entre as ratas fêmeas do que nos ratos machos. Cha et al. (2006) constataram diferenças na sensibilidade do receptor GABA no Sistema Nervoso Central das fêmeas e dos machos sob o uso do álcool.

Em modelos com seres humanos, por sua vez, também são verificadas importantes diferenças entre os sexos, tais como: mulheres são metabolicamente menos tolerantes ao álcool (Epstein et al., 2007), desenvolvem problemas decorrentes do uso mais rapidamente (Mann et al., 2005) embora iniciem o consumo alcoólico mais tardiamente do que os homens. As mulheres apresentaram sua primeira internação psiquiátrica quatro anos e os homens oito anos após o início do consumo regular do álcool (Elbreder, Laranjeira, Siqueira, \& Barbosa, 2008).

Investigações sobre a ação do álcool nas estruturas cerebrais de homens e mulheres usuários crônicos de álcool constataram que os danos cerebrais causados pelo uso do álcool, tais como, atrofia e consequientes perdas neuronais, eram detectadas mais cedo nas mulheres (Hommer, 2003; Mann et al., 2005; Prendergast, 2004). Corroborando estes dados, estudo desenvolvido por Schweinsburg et al. (2003), constatou que as mulheres apresentam perdas mais significativas da substância cinzenta se comparado aos homens.

Outro dado importante envolvendo as diferenças entre os sexos refere-se ao tratamento, estudos constatam que são as mulheres que respondem melhor ao tratamento sendo capazes de se recuperar dos efeitos destrutivos do álcool mais prontamente se comparado aos homens. Como mostra a pesquisa realizada por Satre, Blow, Chi e Weisner (2007), foi constatada diferença significativa entre os sexos quanto à resposta ao tratamento, 76\% das mulheres apresentaram-se abstêmias nos primeiros trinta dias de tratamento, em contra partida, nos homens a porcentagem foi de 54,2.

Em concordância com estes dados, verificou-se através de estudo com uma mostra de 50 homens e 10 mulheres submetidos a tratamento para o uso do álcool, que, após 28 dias de abstinência, as alterações cerebrais foram mais prevalentes no sexo masculino (68\%) comparado ao sexo feminino (20\%), demonstrando melhor resposta das mulheres ao tratamento (Pallavicini et al., 2002). Importante ressaltar que a amostra do referido estudo pode ter comprometido comparações. De todo modo, os dados comprovam a necessidade de estudos que possam investigar de maneira mais precisa o efeito psicoativo do álcool em ambos os sexos.

No sexo feminino, as áreas mais afetadas pelo uso do álcool são as subcorticais como: sistema límbico (emoções); o hipocampo e a amígdala, envolvidos com a memória (Devaud et at., 2003). Dessa forma, as mulheres usuárias de álcool têm prejuízos na memória, maior índice de depressão e risco de suicídio, quando comparadas aos homens (Brady \& Randall, 1999; Graham, Massak, Demers,
\& Rehm, 2007; Pettinati, Pierce, Wolf, Rukstalis, \& O’Brien, 1997; Zaleski, Morato, Silva, \& Lemos, 2004).

No sexo masculino, por sua vez, verifica-se que as áreas mais afetadas pelo uso do álcool são as corticais, especialmente as áreas pré-frontais, envolvidas no raciocínio, no julgamento de valor, na resolução de problemas (Rosenbloom et al., 2005), resultando em alterações de conduta como aumento da impulsividade e agressividade. Sendo, portanto, um aspecto relevante a ser considerado na compreensão dos elevados índices de criminalidade nos homens, usuários de álcool, envolvidos em comportamentos de risco e/ou práticas violentas (Nolen-Hoeksema \& Hilt, 2006).

Associado aos prejuízos cognitivos ocasionados pelo uso prolongado do álcool e seus feitos tóxicos, constata-se que a substância pode desencadear e agravar quadros psiquiátricos severos: transtornos de ansiedade (Kushner, Sher, \& Erickson, 1999) de humor, de conduta (Baldassano, 2006; Corrigan \& Watson, 2005; Enoch, 2003; Oscar-Berman et al., 1997; Salloum, Cornelius, Mezzich, \& Kirisci, 2002) e psicóticos (Marín-León et al., 2007; Zaleski et al., 2004).

Indivíduos que apresentam depressão associada à dependência do álcool, têm maior risco de cometer suicídio (Sher, 2006), sugerindo que as mulheres são mais vulneráveis para desenvolverem a auto-agressão. O uso do álcool no Transtorno do Humor Bipolar, agrava os sintomas maníacos e resulta em maior risco de labilidade emocional, comportamentos impulsivos, violência, e abuso de outras drogas (Salloum et al., 2002). Usuários de substâncias com outros quadros psiquiátricos associados têm risco elevado de desenvolver comportamentos violentos (Corrigan \& Watson, 2005). Em concordância com estes resultados, demonstrou-se que dos 64 usuários do álcool, entre homens e mulheres, selecionados para uma pesquisa, 60\% apresentaram pelo menos um quadro psiquiátrico adicional (Rosenbloom et al., 2005). Em decorrência da alta prevalência de comorbidades no uso do álcool, o tratamento torna-se ainda mais complicado (Enoch, 2003).

\section{Neurobiologia da Violência}

Para maior compreensão sobre a ação do álcool no comportamento violento, do ponto de vista neurobiológico, é importante conhecer os mecanismos envolvidos no comportamento violento. Modelos pré-clínicos, assim como, modelos clínicos: pacientes psiquiátricos com histórico de comportamentos agressivos ou violentos comparados a indivíduos normais são fontes importantes para verificar o papel da serotonina na regulação da agressividade (Liu \& Wuerker, 2005).

Nas últimas décadas, nota-se a relevância da neurotransmissão serotoninérgica na modulação da conduta agressiva ou violenta (Dougherty, Moeller, Bjork, \& Marsh, 1999; Moffitt et al., 1998) especialmente, quando associada à impulsividade. Taxas elevadas de comportamentos impulsivos e violentos e baixa produção de serotonina foram associados ao uso crônico do álcool (Badawy, 2003; 
Johansson et al., 1999; Sher, 2006).

Há um número expressivo de estudos que tratam especialmente sobre a redução da atividade da serotonina em indivíduos com traços agressivos e impulsivos (Sher, 2006), evidenciando que uma anormalidade do metabolismo deste neurotransmissor pode contribuir para maior predis-posição no desenvolvimento desse comportamento. Corroborando estes dados, evidências oriundas de estudos com diferentes metodologias (estudos pot-mortem; estudos de concentrações liquóricas do ácido 5-hidroxi-indolacético (5-HIAA), metabólito da serotonina; estudos neuroendocrinológicos), revelam que anormalidades no sistema serotoninérgico estariam associadas aos comportamento suicida e com características psicobiológicas como: a impulsividade e a agressividade (Correa, 2004).

A partir de um trabalho de revisão com artigos publicados entre os anos 1994 e 1999, verificou-se que em seres humanos, sinais clínicos e laboratoriais de baixa atividade serotoninérgica relacionam-se com agressividade, impulsividade, depressão, suicídio e alcoolismo de início precoce. A depressão e o alcoolismo são os diagnósticos psiquiátricos mais comuns no suicídio, sendo que, dentre os usuários crônicos do álcool, a baixa atividade serotoninérgica esteve relacionada com o suicídio impulsivo (Moreira, 2001).

Além do importante envolvimento da serotonina, estudos clínicos demonstram que medicamentos com ação em sistemas neuroquímicos como: o dopaminérgico (Sher, 2006), o gabaérgico (GABA) e o noradrenérgico estão envolvidos na regulação da agressividade, demonstrando importante associação também desses sistemas no controle do comportamento agressivo.

\section{Considerações Finais}

A realização do presente estudo possibilitou identificar uma extensa e crescente literatura referente ao uso nocivo e/ou crônico do álcool, bem como, aos prejuízos individuais e sociais a eles associados; dentre os principais prejuízos, verificou-se, estreita relação entre o consumo alcoólico e a violência. Além dos homens, as mulheres também passam a ser população de atenção e investigação pela comunidade científica, uma vez que, constatam-se diferenças importantes entre homens e mulheres quanto à ação psicoativa do álcool, sendo as mulheres mais vulneráveis aos efeitos deletérios da substância.

As diferenças entre os sexos quanto à ação do álcool no organismo são conhecidas, entretanto, estudos que relacionem a ação psicoativa do álcool com os mecanismos neurobiológicos envolvidos no comportamento violento de homens e mulheres, bem como, as diferenças existentes entre ambos os sexos, são escassos. Além disso, os estudos que se referem ao uso do álcool e a violência, em sua maioria, são reflexões sobre as diferenças de gênero e não de sexo. Tais aspectos caracterizaram tanto a relevância do estudo por tratar-se de um assunto ainda pouco explorado, como também, as limitações do mesmo.

Estudos futuros que focalizem as diferenças existentes entre os sexos quanto aos mecanismos neurobiológicos envolvidos na ação psicoativa do álcool sob o comportamento de homens e mulheres são necessários, especialmente, quando se refere ao comportamento violento e seu impacto na qualidade de vida destas pessoas e na sociedade em geral. Por fim, compreender as diferenças entre os sexos analisando as formas de violência é de fundamental importância para desenvolver planos de tratamento mais eficazes, adequados à população feminina e masculina.

\section{Referências}

Abrahams, N., Jewkes, R., Laubscher, R., \& Hoffman, M. (2006). Intimate partner violence: Prevalence and risk factors for men in Cape Town, South Africa. Violence and Victims, 21(2), 247264.

Adeodato, V. G., Carvalho, R. R., Siqueira, V. R., \& Souza, F. G. M. (2005). Qualidade de vida e depressão em mulheres vítimas de seus parceiros. Revista de Saúde Pública, 39(1), 108-113.

Amen, D. G., Stubblefield, M., Carmicheal, B., \& Thisted, R. (1999). Brain SPECT findings and aggressiveness. Annual Clinical Psychiatry, 8(3), 129-137.

Augengraun, M., Wilson, T. E., \& Allister, L. (2001). Domestic violence reported by women attending a sexually transmitted desease clinic. Jornal American Sexually Transmitted Disease Association, 28,143-146.

Badawy, A. A. (2003). Alcohol and violence and the possible role of serotonin. Criminal Behaviour Mental Health, 13(1), 31-44.

Baldassano, C. F. (2006). Illness course, comorbidity, gender, and suicidality in patients with bipolar disorder. Journal of Clinical Psychiatry, 67(11), 8-11.

Bartels, C., Kunert, H. J., Stawicki, S., Kroner-Herwig, B., Ehrenreich, H., \& Krampe, H. N. (2007). Recovery of hippocampus-related functions in chronic alcoholics during monitored long-term abstinence. Alcohol and Alcoholism, 42(2), 92-102.

Bates, M. (1997). Stability of neuropsychological assessments early in alcoholism treatment. Journal of studies on alcohol, 58(6), 617-622.

Bonomo, Y., Coffey, C., Wolfe, R., Lynskey, M., Bowes, G., \& Patton, G. (2001). Adverse outcomes of alcohol use in adolescents. Addiction, 96(10), 1485-1496.

Borders, A., Barnwell, S. S., \& Earleywine, M. (2007). Alcoholaggression expectancies and dispositional rumination moderate the effect of alcohol consumption on alcohol-related aggression and hostility. Aggressive Behavior, 33(4), 327-338.

Brady, K., \& Randall, C. (1999). Gender differences in substance use disorders. Psychiatric Clinics of North America, 22(2), 241-252.

Brown, S. A., Tapert, S. F., \& Brown, G. G. (2001). Measurement of brain dysfunction in alcohol-dependent young women. Alcohol Clinical Experimental Research, 25, 236-245.

Brown, S. A., Tapert, S. F., Granholm, E., \& Delis, D. C. (2000). Neurocognitive functioning of adolescents: Effects of protracted alcohol use. Alcoholism: Clinical \& Experimental Research, 24(2), 164-171.

Brun, A., \& Andersson, J. (2001). Frontal dysfunction and frontal cortical synapse loss in alcoholism: The main cause of alcohol dementia? Dementia Geriatric Cognitive Disorder, 12(4), 289-294.

Cha, Y. M., Li, Q., Wilson, W. A., \& Swartzwelder H. S. (2006). 
Sedative and GABAergic effects of ethanol on male and female rats. Alcoholism: Clinical and Experiental Research, 30(1), 113-118.

Chalub, M., \& Telles, L. E. B. (2006). Álcool, drogas e crime. Revista Brasileira de Psiquiatria, 28(2), 69-73.

Chavez, K. A. P., O’brien, B., \& Pillon, S. C. (2005). Uso de drogas e comportamentos de risco no contexto de uma comunidade universitária. Revista Latino-Americana de Enfermagem, 13(2), 1194-1200.

Chermack, S. T., \& Giancola, P. R. (1997). The relation between alcohol and aggression: An integrated biopsychosocial conceptualization. Clinical Psychology Review, 17(6), 621649.

Chermack, S. T., Wryobeck, J. M., Walton, M. A., \& Blow, F. C. (2006). Distal and proximal factors related to aggression severity among patients in substance abuse treatment: Family history, alcohol use and expectancies. Addictive Behaviors, 31(5), 845-858.

Clark, D. B., Lynch, K. G., Donovan, J. E., \& Block, G. D. (2001). Health problems in adolescents with alcohol use disorders: Self-report, liver injury, and physical examination findings and correlates. Alcoholism: Clinical \& Experimental Research, 25(9), 1350-1359.

Conner, K. R., Cox, C., Duberstein, P. R., Tian, L., Nisbet, P. A., \& Conwell, Y. (2001). Violence, alcohol, and completed suicide: A case-control study. American Journal of Psychiatry, 158(10), 1701-1705.

Correa, H. (2004). Suicídio e impulsividade: Pesquisas sobre o papel do sistema serotoninérgico. Biological Psyquiatry, 12(1), 19-22.

Corrigan, P. W., \& Watson, A. C. (2005). Findings from the National Comorbidity Survey on the frequency of violent behavior in individuals with psychiatric disorders. Psychiatry Research, 136(2/3), 153-162.

Cunha, P. J., \& Novaes, M. A. (2004). Avaliação neurocognitiva no abuso e dependência do álcool: Implicações para o tratamento. Revista Brasileira de Psiquiatria, 26(1), 23-27.

Devaud, L. L., Alele, P., \& Ritu, C. (2003). Sex differences in the central nervous system action of ethanol. Critical Reviews Neurobiology, 15(1), 41-59.

Dick, D. M., \& Bierut, L. J. (2006). The genetics of alcohol dependence. Current Psychiatry Reports, 8(2), 151-157.

Dougherty, D. M., Moeller, F. G., Bjork, J. M., \& Marsh, D. M. (1999). Plasma L-tryptophan depletion and aggression. Advances in Experimental Medicine and Biology, 467, 57-65.

Eckardt, M. J., File, S. E., Gessa, G. L., Grant, K. A., Guerri, C., Hoffman, P. L., et al. (1998). Effects of moderate alcohol consumption on the central nervous system. Alcoholism: Clinical and Experimental Research, 22(5), 998-1040.

Edelstein, S. L., Kritz-Silverstein, D., \& Barrett-Connor, E. (1998). Prospective association of smoking and alcohol use with cognitive function in an elderly cohort. Journal of Womens Health, 7(10), 1271-1281.

Elbreder, M. F., Laranjeira, R., Siqueira, M. M., \& Barbosa, D. A. (2008). Perfil de mulheres usuárias de álcool em ambulatório especializado em dependência química. Jornal Brasileiro de Psiquiatria, 57(1), 9-15.

Enoch, M. A. (2003). Pharmacogenomics of alcohol response and addiction. American Journal of Pharmacogenomics, 3(4), 217232.

Epstein, E. E., Fischer-Elber, K., \& Al-Otaiba, Z. (2007). Women, aging, and alcohol use disorders. Journal of Women \& Aging, 19(1), 31-48.

Fontes, A., Figlie, N. B., \& Laranjeira, R. (2006). O comporta- mento de beber entre dependentes de álcool: Estudo de seguimento. Revista de Psiquiatria Clínica, 33(6) 304-312.

Galera, S. A. F., Roldán, B. M. C., \& O’brien, B. (2005). Women living in a drug (and violence) context: The maternal role [Special issue]. Revista Latino-Americana de Enfermagem, 13(2), 1142-1147.

Glazkov, V. A., \& Sviderskaia, N. E. (2006). Gender differences in chronic alcoholism: EEG analysis. Zh Vyssh Nerv Deiat Im I P Pavlova, 56(5), 597-602.

Graham, K., Massak, A., Demers, A., \& Rehm, J. (2007). Does the association between alcohol consumption and depression depend on how they are measured? Alcoholism: Clinical and Experimental Research, 31(1), 78-88.

Grant, J. D., Scherrer, J. F., Lynskey, M. T., Lyon, M. J., Eisen, S. A., Tsuang, W. R., et al. (2006). Adolescent alcohol use is a risk factor for adult alcohol and drug dependence: Evidence from a twin design. Psychological Medicine, 36(1), 109-118.

Gudlaugsdottir, G. R., Vilhjalmsson, R., Kristjansdottir, G., Jacobsen, R., \& Meyrowitsch, D. (2004). Violent behaviour among adolescents in Iceland: A national survey. International Journal of Epidemiology, 33(5), 1046-1051.

Hasegawa, M., Bessho, Y., Hosoya, T., \& Deguchi Y. (2005). Prevalence of intimate partner violence and related factors in a local city in Japan. Japanese Journal of Public Health, 52(5), 411-421.

Hernandéz-Ávila, C. A., Rounsaville, B. J., \& Kranzler, H. R. (2004). Opioid, cannabis and alcohol dependent women show more rapid progression to substance abuse treatment. Drug and Alcohol Dependence, 74, 265-272.

Hommer, D. W. (2003). Male and female sensitivity to alcoholinduced brain damage. Alcohol Research \& Health, 27(2), 181185.

Johansson, A. K., Bergvall, A. H., \& Hansen, S. (1999). Behavioral disinhibition following basal forebrain excitotoxin lesions: Alcohol consumption, defensive aggression, impulsivity and serotonin levels. Behaviour Brain Research, 102(1/2), 17-29.

Kirkcaldy, B. D., Siefen, G. R., Urkin, J., \& Merrick, J. (2006). Risk factors for suicidal behavior in adolescents. Minerva Pediatrica, 58(5), 443-450.

Kushner, M. G., Sher, K. J., \& Erickson D. J. (1999). Prospective analysis of the relation between DSM-III anxiety disorders and alcohol use disorders. The American Journal Psychiatry, 156, 723-732.

Laranjeira, R. (2004). Apresentação: Álcool: Da saúde pública à comorbidade psiquiátrica. Revista Brasileira de Psiquiatria, 26(1), 1-2.

Laranjeira, R., \& Romano, M. (2004). Brazilian consensus on public policies on alcohol. Revista Brasileira de Psiquiatria, 26(1), 68-77.

Lavine, R. (1997). Psychopharmacological treatment of aggression and violence in the substance using population. Journal of Psychoactive Drugs, 29(4), 321-329.

Liu, J., \& Wuerker, A. (2005). Biosocial bases of aggressive and violent behavior: Implications for nursing studies. International Journal of Nursing Studies, 42(2), 229-241.

Mack, A. H., \& Frances, R. J. (2003). Treatment of alcohol use disorders in adolescents. Journal of Psychiatric Practice, 9(3), 195-208.

Mann, K., Ackermann, K., Croissant, B., Mundle, G., Nakovics, H., \& Diehl, A. (2005). Neuroimaging of gender differences in alcohol dependence: Are women more vulnerable? Alcoholism: Clinical \& Experimental Research, 29(5), 896-901. 
de Almeida, R. M. M., Pasa, G. G. \& Scheffer, M. (2009). Álcool e Violência em Homens e Mulheres

Marín-León, L., Oliveira, H. B., \& Botega, N. J. (2007). Mortalidade por dependência de álcool no Brasil: 1998 - 2002. Psicologia em Estudo, 12(1), 115-121.

Marques, A. C. P. R., \& Cruz, M. S. (2000). O adolescente e o uso de drogas. Revista Brasileira de Psiquiatria, 22(2), 32-36.

Martin, S. E., \& Bryant, K. (2001). Gender differences in the association of alcohol intoxication and illicit drug abuse among persons arrested for violent and property offenses. Journal of Substance Abuse, 13(4), 563-581.

Meloni, J. N., \& Laranjeira, R. (2004). Custo social e de saúde do uso do álcool. Revista Brasileira de Psiquiatria, 26(1), 7-10.

Mitchell, J. M., Fields, H. L., D'Esposito, M., \& Boettiger, C. A. (2005). Impulsive responding in alcoholics. Alcoholism: Clinical \& Experimental Research, 29(12), 2158-2169.

Moffitt, T. E., Brammer, G. L., Caspi, A., Fawcett, J. P., Raleigh, M., Yuwiler, A., et al. (1998). Whole blood serotonin relates to violence in an epidemiological study. Biological Psychiatry, 43(6), 446-457.

Monnot, M., Nixon, S., Lovallo, W., \& Ross, E. (2001). Altered emotional perception in alcoholics: Deficits in affective prosody comprehension. Alcoholism: Clinical and Experimental Research, 25(3), 362-369.

Moreira, C. N. (2001). Serotonina e comportamento agressivoimpulsivo. Biological Psyquiatry, 9(1), 3-11.

Nakahara, T., Hirano, M., Uchimura, H., Shirali, S., Martin, C. R., Bonner, A. B., et al. (2002). Chronic alcohol feeding and its influence on c-Fos and heat shock protein-70 gene expression in different brain regions of male and female rats. Metabolism, 51(12), 1562-1568.

Nobrega, M. P. S., \& Oliveira, E. M. (2005). Mulheres usuárias de álcool: Análise qualitativa. Revista de Saúde Pública, 39(5), 816-823.

Nolen-Hoeksema, S., \& Hilt, L. (2006). Possible contributors to the gender differences in alcohol use and problems. The Journal of General Psychology, 133(4), 357-374.

Organização Mundial da Saúde. (2004). Neurociências: Consumo e dependência de substâncias psicoativas: Resumo. Genebra, Suíça: Autor.

Oscar-Berman, M., Shagrin, B., Evert, D. L., \& Epstein, C. (1997). Impairments of brain and behavior: The neurological effects of alcohol. Alcohol Health \& Research World, 21(1), 65-75.

Pallavicini, G. J., Massardo, V. T., Arancibia, S. P., González, E. P., Cumsille, G. F., \& Padilla, P. P. (2002). Behavior of brain perfusion with SPECT tomography $99 \mathrm{mTc}$ ethylene dicysteine (ECD) in alcohol and cocaine dependents during abstinence. Revista Española de Medicina Nuclear, 21(6), 410-416.

Parker, R. N., \& Auerhahn, K. (1998). Alcohol, drugs, and violence. Annual Review of Sociology, 24 (1), 291-311.

Pechansky, F., Szobot, C. M., \& Scivoletto, S. (2004). Uso de álcool entre adolescentes: Conceitos, características epidemiológicas e fatores etiopatogênicos. Revista Brasileira de Psiquiatria, 26(1), 14-17.

Pettinati, H. M., Pierce, J. D., Wolf, A. L., Rukstalis, M. R., \& O'Brien, C. P. (1997). Gender differences in comorbidly depressed alcohol-dependent outpatients. Alcoholism-Clinical and Experimental Research, 21(9), 1742-1746.

Pettinati, H., \& Rabinowitz, A. (2005). Recent advances in the treatment of alcoholism. Clinical Neuroscience Research, 5(2/ 4), 151-159.

Pfefferbaum, A., Adalsteinsson, E., \& Sullivan, E. (2006). Dysmorphology and microstructural degradation of the corpus callosum: Interaction of age and alcoholism. Neurobiology of Aging, 27(7), 994-1009.
Pillay, A. L., Van der Veen, M. B., \& Wassenaar, D. R. (2001). Non-fatal suicidal behaviour in women: The role of spousal substance abuse and marital violence. South African Medical Journal, 91(5), 429-432.

Pillon, S. C., O'Brien, B., \& Chavez, K. A. P. (2005). The relationship between drugs use and risk behaviors in Brazilian university students. Revista Latino-Americana de Enfermagem, 13(2), 1169-1176.

Prendergast, M. A. (2004). Do women possess a unique susceptibility to the neurotoxic effects of alcohol? Journal of American Medicine Womens Association, 59(3), 225-227.

Rabello, P. M., \& Caldas Junior, A. F. (2007). Violência contra a mulher, coesão familiar e drogas. Revista de Saúde Pública, 41(6), 970-978.

Ramisetty-Mikler, S., Goebert, D., Nishimura, S., \& Caetano, R. (2006). Dating violence victimization: Associated drinking and sexual risk behaviors of Asian. Native Hawaiian, and Caucasian high school students in Hawaii. Journal of School Health, 76(8), 423-429.

Ramos-Lira, L., Saltijeral-Méndez, M. T., Romero-Mendoza, M., Caballero-Gutiérrez, M. A., \& Martínez-Vélez, N. A. (2001). Violencia sexual y problemas asociados en una muestra de usuarias de un centro de salud. Salud Pública (México), 43(3), 182-191.

Rosenbloom, M. J., O'Reilly, A., Sassoon, S. A., Sullivan, E. V., \& Pfefferbaum, A. (2005). Persistent cognitive deficits in community-treated alcoholic men and women volunteering for research: Limited contribution from psychiatric comorbidity. Journal of Studies on Alcohol, 66(2), 254-265.

Roy, K., Parker, G., Mitchell, P., \& Wilhelm, K. (2001). Depression and smoking: Examining correlates in a subset of depressed patients. Australian and New Zealand Journal of Psychiatry, 35(3), 329-335.

Salloum, I. M., Cornelius, J. R., Mezzich, J. E., \& Kirisci, L. (2002). Impact of concurrent alcohol misuse on symptom presentation of acute mania at initial evaluation. Bipolar Disorder, 4(6), 418-421.

Saletu-Zyhlarz, G. M., Arnold, O., Anderer, P., Oberndorfer, S., Walter, H., Lesch, O. M., et al. (2004). Differences in brain function between relapsing and abstaining alcohol-dependent patients, evaluated by eeg mapping. Alcohol \& Alcoholism, 39(3), 233-240.

Satre, D. D., Blow, F. C., Chi, F. W., \& Weisner C. (2007). Differences in seven-year alcohol and drug treatment outcomes among older adults. American Journal on Addictions, 16(3), 216-221.

Schraiber, L. B., D’Oliveira, A. F. P. L., Franca-Junior, I., \& Pinho, A. A. (2002). Violência contra a mulher: Estudo em uma unidade de atenção primária à saúde. Revista de Saúde Pública, 36(4), 470-477.

Schweinsburg, B. C., Alhassoon, O. M., Taylor, M. J., Gonzalez, R., Videen, J. S., Brown, G. G., et al. (2003). Effects of alcoholism and gender on brain metabolism. American Journal Psychiatry, 160(6), 1180-1183.

Sher, L. (2006). Alcohol and suicide: Neurobiological and clinical aspects. Scientific World Journal, 6, 700-706.

Simão, M. O., Kerr-Corrêa, F., Dalben, I., \& Smaira, S. I. (2002). Alcoholic women and men: A comparative study of social and familial aspects and outcome. Revista Brasileira de Psiquiatria, 24(3), 121-129.

Siqueira, M. M. (2004). Debate sobre o artigo de Delma Pessanha Neves. Caderno de Saúde Pública, 20(1), 15-16. 
Thompson, M. P., \& Kingree, J. B. (2004). The role of alcohol use in intimate partner violence and non-intimate partner violence. Violence Victims, 19(1), 63-74.

Valdez-Santiago, R., Juárez-Ramírez, C., Salgado-de Snyder, V. N., Agoff, C., Avila-Burgos, L., \& Híjar, M. C. (2006). Violencia de género y otros factores asociados a la salud emocional de las usuarias del sector salud en México. Salud Pública de México, 48(2), 250-258.

Zaleski, M., Morato, G. S., Silva, V. A., \& Lemos, T. (2004). Aspectos neurofarmacológicos do uso crônico e da Síndrome de Abstinência do Álcool. Revista Brasileira de Psiquiatria, 26(1), 40-42.

Zilberman, M. L., \& Blume, S. B. (2005). Violência doméstica, abuso de álcool e substâncias psicoativas. Revista Brasileira de Psiquiatria, 27(Supl. 2), s51-s55.

Vaiz Bonifaz, R. G., \& Nakano, M. S. (2004). La violencia intrafamiliar, el uso de drogas en la pareja, desde la perspectiva de la mujer maltratada [Special issue]. Revista Latino-Americana de Enfermagem, 12, 433-438.

Watzke, S., Ullrich, S., \& Marneros, A. (2006). Gender and violence-related prevalence of mental disorders in prisoners. European Archives of Psychiatry and Clinical Neuroscience, 256(7), 414-421. 